\title{
Military applications of Hyperspectral Imagery
}

\author{
Briottet X. ${ }^{1}$, Boucher Y. ${ }^{1}$, Dimmeler A. ${ }^{2}$, Malaplate A. ${ }^{2}$, Cini A. ${ }^{3}$, Diani M. ${ }^{3}$, Bekman H. ${ }^{4}$, \\ Schwering P. ${ }^{4}$, Skauli T. ${ }^{5}$, Kasen I. ${ }^{5}$, Renhorn I. ${ }^{6}$, Klasén L. ${ }^{6}$, Gilmore M. ${ }^{7}$, Oxford D. ${ }^{7}$ \\ ${ }^{1}$ : ONERA, France \\ 2: FGAN, Germany \\ 3 : CISAM, Italy \\ ${ }^{4}:$ TNO, The Netherlands \\ ${ }^{5}$ : FFI, Norway \\ ${ }^{6}$ : FOI, Sweden \\ 7. DSTL, United-Kingdom
}

\begin{abstract}
:
Optical imaging, including infrared imaging, generally has many important applications, both civilian and military. In recent years, technological advances have made multi- and hyperspectral imaging a viable technology in many demanding military application areas. The aim of the CEPA JP 8.10 program has been to evaluate the potential benefit of spectral imaging techniques in tactical military applications. This unclassified executive summary describes the activities in the program and outlines some of the results. More specific results are given in classified reports and presentations.

The JP 8.10 program started in March 2002 and ended in February 2005. The participating nations were France, Germany, Italy, Netherlands, Norway, Sweden and United-Kingdom, each with a contribution of 2 man-years per year. Essential objectives of the program were to:

- analyze the available spectral information in the optronic landscape from visible to infrared;

- analyze the operational utility of multi- and hyperspectral imaging for detection, recognition and identification of targets, including low-signature targets;

- identify applications where spectral imaging can provide a strong gain in performance;

- propose technical recommendations of future spectral imaging systems and critical components.

Finally, a stated objective of the JP 8.10 program is to "ensure the proper link with the image processing community".

The presentation is organized as follows. In a first step, the two trials (Pirrene and Kvarn) are presented including a summary of the acquired optical properties of the different landscape materials and of the spectral images. Then, a phenomenology study is conducted analyzing the spectral behavior of the optical properties, understanding the signal at the sensor and, by processing spectroradiometric measurements evaluating the potential to discriminate spectral signatures.

Cameo-Sim simulation software is presented including first validation results and the generation of spectral synthetic images. Results obtained on measured and synthetic images are shown and discussed with reference to two main classes of image processing tasks: anomaly detection and signature based target detection. Furthermore, preliminary works on band selection are also presented which aim to optimize the spectral configuration of an image sensor. Finally, the main conclusions of the WEAG program CEPA JP8.10 are given.
\end{abstract}

Keywords: Hyperspectral imagery, modeling, phenomenology, anomaly detection, signature based detection, signature, band selection

\section{Introduction}

Optical imaging, including infrared imaging, generally has many important applications, both civilian and military. In recent years, technological advances have made multi- and hyperspectral imaging a viable technology in many demanding military application areas. The aim of the CEPA JP 8.10 program has been to evaluate the potential benefit of spectral imaging techniques in tactical military applications.

The JP 8.10 program started in March 2002 and ended in February 2005. The participating nations were France, Germany, Italy, Netherlands, Norway, Sweden and United-Kingdom. Essential objectives of the program were to:

- analyze the available spectral information in the optronic landscape from visible to infrared;

Targets and Backgrounds XII: Characterization and Representation, edited by Wendell R. Watkins, Dieter Clement, Proc. of SPIE Vol. 6239

62390B, (2006) - 0277-786X/06/\$15 - doi: 10.1117/12.672030

Proc. of SPIE Vol. 6239 62390B-1 
- analyze the operational utility of multi- and hyperspectral imaging for detection, recognition and identification of targets, including low-signature targets;

- identify applications where spectral imaging can provide a strong gain in performance;

- propose technical recommendations of future spectral imaging systems and critical components.

Finally, a stated objective of the JP 8.10 program is to "ensure the proper link with the image processing community".

The document is organized as follows. In a first step, the two trials (Pirrene and Kvarn) are presented in $\$ 2$ including a summary of the acquired optical properties of the different landscape materials and of the spectral images. Then, a phenomenology study is conducted in $\$ 3$ analyzing the spectral behavior of the optical properties, understanding the signal at the sensor and, by processing spectroradiometric measurements evaluating the potential to discriminate spectral signatures.

In $\S 4$, Cameo-Sim simulation software is presented including first validation results and the generation of spectral synthetic images. The resulting measured and synthetic images are processed and the results shown in $\S 5$. Two main classes of image processing are presented: anomaly detection and signature based detection. Furthermore, preliminary works on band selection are also presented which aim to optimize the spectral configuration of an image sensor. In $\S 6$, the main conclusions of the WEAG program CEPA JP8.10 are given.

\section{Description of the field trials}

\subsection{Background}

Two major field trials have been carried out.

The first field campaign took place in France, May to October, 2002 at the Pirrene site near Toulouse. Its objectives were to validate the simulation codes: Cameo-Sim (UK) and Comanche (Fr), to gather initial ground-to-ground spectral data for spectral processing and to conduct phenomenology studies. In this trial, a variety of instrumentation (3 BOMEM spectroradiometers, 2 portable spectroradiometers, 2 hyperspectral imaging sensors, several multispectral imaging sensors) at ground level were used to characterize a set of panels and other simple objects at short range, including objects with surface properties representative of relevant military targets. The instrumentation covered optical wavelengths from the visible to the long wave infrared. Measurements were made over a period of several months at different time scales (hourly, daily and monthly bases) and under a wide range of weather and illumination conditions, in order to characterize the natural scene variability.

The Kvarn campaign took place at Prästtomta Live Fire Range in Kvarn, Linköping, Sweden, May 26 to June 14, 2003. The purpose of the Kvarn trial was to find new ways of looking at military targets and backgrounds using multispectral and hyperspectral sensors. This trial employed a large number of military targets including vehicles, decoys and camouflage in a forest/rural background, as illustrated in Figure 1. A variety of tactical scenarios were tested, such as:

- $\quad$ detection of targets, including targets in varying degrees of hide and camouflage;

- $\quad$ classification of targets, rejection of decoys and civilian-military discrimination.

At Kvarn, image data were recorded in the entire optical wavelength domain using a variety of imaging sensors including multi- and hyperspectral cameras, a polarimetric camera and several conventional cameras at ground level. Hyperspectral and also high spatial resolution images were recorded from the air by three different aircraft. Extensive ground truth data were collected from the scene (Figure 1). In particular, the optical properties of a variety of materials were recorded and compiled into a highly useful material database (more than 600 spectra have been collected). These data were combined with a detailed geometrical terrain model to produce a high-resolution simulation model for the entire scene. A total of more than 100 people participated at the Kvarn trial. The two week campaign gave information on both diurnal variations and weather dependent variations. The variation in weather, together with the variation in target states, gave the opportunity to study target to background contrasts under varying conditions. 

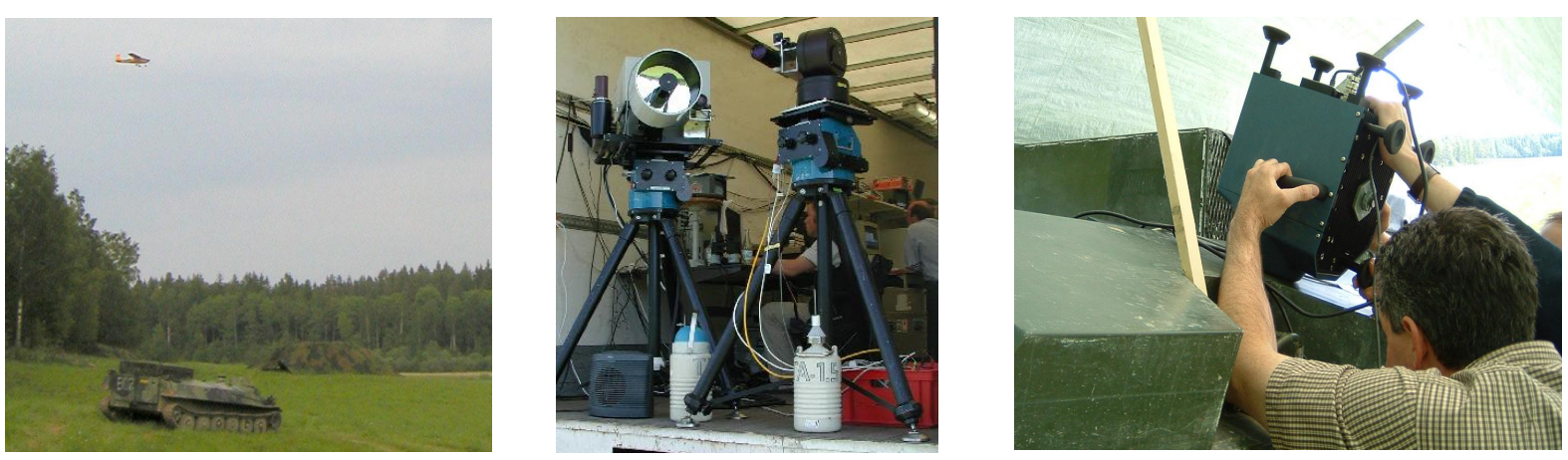

Figure 1: At the Kvarn campaign, a wide range of airborne and ground based sensors recorded data from a large selection of military targets. Leftmost figure: example of a military target, Central figure: Bomem Spectroradiometer (left) and Imspec hyperspectral image sensor (right), Rightmost figure: spectral reflectance measurement on a military target using the SOC 400T.

During these two trials, atmospheric characterizations were continuously conducted using temperature data logger, weather stations, weather forecasts and aerosol measurements.

\subsection{Data description}

\subsubsection{Optical properties}

For this CEPA8 program it was essential to know the optical properties of all the materials, which were present at the field trials, as these data were basic inputs for other works in this program. The optical properties data were taken for the whole optical domain with state-of-the-art instrumentations.. Directional Hemispherical Reflectance (DHR) were measured from the UV $(0.3 \mu \mathrm{m})$ up to the LWIR $(14 \mu \mathrm{m}$ and some even up to $25 \mu \mathrm{m})$, the Bidirectional Reflectance Distribution Function (BRDF) data, in broad-band, were taken in the MWIR $(3-5 \mu \mathrm{m})$ and LWIR $(8-12 \mu \mathrm{m})$ and some spectral BRDF between $0.4 \mu \mathrm{m}$ and $2.5 \mu \mathrm{m}$. To be able to generate a large data base, an inter-comparison process had been performed in a Round-Robin test involving all our facilities. In consequence, it was ensured, that each country contributed with the same quality of data to the data base. The list of used instrumentations is given in Table 1 .

Table 1: List of on ground sensors to measure the optical properties

\begin{tabular}{|l|c|c|}
\hline Sensor & Spectral Range & Reflectance type \\
\hline ASD FieldSpec & $0.4 \mu \mathrm{m}-2.5 \mu \mathrm{m}$ & Spectral DHR \\
\hline SOC 600 & MWIR, LWIR & BRDF \\
\hline GER 3700+ Chamo & $0.4 \mu \mathrm{m}-2.5 \mu \mathrm{m}$ & Spectral BRDF \\
\hline SOC 400T & $2 \mu \mathrm{m}-14 \mu \mathrm{m}$ & Spectral DHR \\
\hline
\end{tabular}

During the program more than 95 materials were measured in the laboratories and in the field, including diurnal and seasonal variations. These 95 materials can be separated into two categories, 60 man-made materials (paints, foils, canvas, camouflage nets...) and 35 natural background materials (vegetation, soil....). The data were acquired in more than 1300 measurements and were all stored in the data base, MEMOIRES.

\subsubsection{Image data}

During the Pirrene trial in July 2003, images were acquired under different weather conditions with horizontal viewing at short range both in the visible (ASI hyperspectral image sensor) and infrared (multispectral cameras) domains.

But most images were acquired during the Kvarn experiment with a broad range of sensors, ranging from single band sensors to hyperspectral sensors. These sensors provide combinations of high spatial and spectral resolution of background and targets of interest. A list of image sensors is given in Table 2. This large image data set is thus available over a rural landscape including different types of military targets in different status (engine on / off, open / semi-hide / deep, several weather conditions, different times in a day...) with on-ground and airborne viewing. 
Table 2: List of image sensors used during Kvarn trial

\begin{tabular}{|l|c|c|c|}
\hline Sensor & Type & Spectral Range $(\boldsymbol{\mu m})$ & $\begin{array}{c}\text { Airborne / On- } \\
\text { Ground }\end{array}$ \\
\hline Pelican & High spatial resolution & Visible & On-Ground \\
\hline Timbre-Poste & High spatial resolution & MWIR & On-Ground \\
\hline Hasselblad & High spatial resolution & Visible & Airborne \\
\hline AIM-640Q MW & Broadband & $4-5$ & On-Ground \\
\hline AIM-640Q LW & Broadband & $8-9$ & On-Ground \\
\hline Canon D60 & Multispectral & Visible & On-Ground \\
\hline AIM 4C & Multispectral & $3.4-4.1,4.5-5.3,3.4-5.3,1.2-5.3$ & On-Ground \\
\hline MULTIMIR & Multispectral & $1.55-1.75,2.05-2.45,3.45-4.15,4.55-5.2$ & On-Ground \\
\hline ASI & Hyperspectral & $0.4-0.9$ & $\begin{array}{c}\text { Airborne, On- } \\
\text { Ground }\end{array}$ \\
\hline SOC 700 & Hyperspectral & $0.4-0.9$ & On-Ground \\
\hline MIVIS & Hyperspectral & $0.43-0.83,1.15-1.55,1.99-2.48,8.21-12.70$ & Airborne \\
\hline IMSPEC & Hyperspectral & $0.4-0.9$ & On-Ground \\
\hline REMIDS & Polarization & $3-5$ & On-Ground \\
\hline
\end{tabular}

\section{Phenomenology}

The aims of this study are to understand the constitution of the spectral radiance signal and its behavior versus the environmental parameters, to analyze the spectral optical properties of materials and to assess the separability of the materials.

This study has been carried out considering the whole optical domain, with an emphasis on the MWIR and LWIR domain because of the large amount of available data. Moreover, this domain has broader applications for defense purpose and needs more studies because of the complexity of the signal constitution.

The data set used comes from the two campaigns (Pirrene and Kvarn) including spectral reflectance and at sensor spectral radiance in all the optical bands at various environmental conditions.

A first analysis was performed to understand the different phenomena and their relative contributions to the total at sensor signal as a function of the wavelength, using the Comanche radiative code.

Then, the spectral behavior of the collected reflectance spectra from different materials in different state was interpreted. The main result points out the impact on the spectral signature as the vegetation dies (Figure 2).

Furthermore, the spectral target/background contrast analysis has been carried out with the PIRRENE and KVARN BOMEM data set. Its dependency versus environmental parameters appears complex, and is mainly ruled by temperature aspects. Nevertheless, general trends have been found and these results shows that the spectral contrast in IR is mainly dependent on time of day: low contrast appears at early morning or at late evening, when temperature is low, and when relative humidity is high.

In a last step, the potential capability of discriminating different materials using discriminant analysis has been evaluated. In the reflective domain, shapes of radiance spectra show clear differences that can be directly used for discrimination without a complete atmospheric correction. On the other hand, in MWIR and LWIR domain, using radiance spectra, no convincing results can be obtained without temperature/emissivity separation algorithm (TES). This discrimination is possible using their reflectance spectra. Thus, as a conclusion, atmospheric compensation and emissivity/temperature separation are required to discriminate materials in the infrared domain.

\section{Modeling and simulation}

The objective of this effort was to demonstrate that the application of multi- and hyperspectral technology can be realistically represented through modeling. Hence, the majority of the work performed in order to achieve this objective was directed towards validating the electro-optic imaging simulation tool, Cameo-Sim. Cameo-Sim is a modular software suite that has been developed by Insys Limited, under funding from UK MoD, to produce high fidelity synthetic imagery between 0.3 and $20 \mu \mathrm{m}$. Cameo-Sim is a first principles model in that it solves various forms of the radiation transport equation for the natural environment using physically meaningful parameters. A validation methodology for Cameo-Sim was defined and various studies were performed comparing real data, collected at the Kvarn trial, with synthetic data. These included the comparison of primary data, i.e. hyperspectral imagery collected by 
airborne sensors, and secondary data, e.g. surface temperatures and atmospheric fluxes. The studies undertaken so far have shown fairly good comparison between real and synthetic data and, in some cases, gone beyond initial expectations - this is certainly true of the performance of a target detection algorithm where reasonable comparison has been demonstrated for the first time. Overall, progress has been extremely encouraging and the modeling process requirements are better understood e.g. capturing the correct tree density in the modeled scene. This has identified areas where Cameo-Sim may require modifications e.g. capturing the effects of the 3D nature of grass. It is therefore hoped that when these issues are addressed the comparisons will improve.

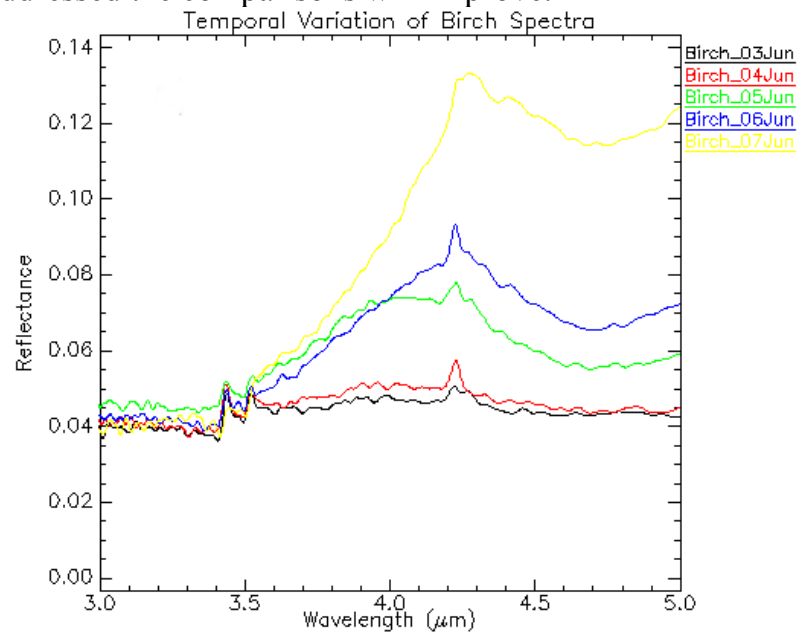

Figure 2: Spectral reflectance of birch: effects of decay modifying the water content in the $4-5 \mu$ m. The birch was cut on the $3^{\text {rd }}$ of March, and its reflectance measured every day during 4 days

\section{Image processing}

\subsection{Spectral image processing}

For use of spectral imaging against military targets, some of the most important types of image processing are anomaly detection, signature-based detection (or spectral recognition) and band selection. These will be discussed in the following. Other types of spectral image processing may also be applied. For example, many civilian applications of spectral imaging aim at estimating quantitative state parameters of the scene, such as the condition of vegetation, and such techniques may also have military applications.

Prior to the information-extracting processing steps, some form of preprocessing is usually applied to the data, for example to apply sensor calibration coefficients or to compensate for effects of platform movement and atmosphere.

The JP 8.10 program has established several capabilities for processing of spectral images. The target detection work has mostly been based on already existing algorithms. It can be noted, however, that the performance of an algorithm depends significantly on the implementation and adaptation for the task at hand. By covering the entire signal chain from scene through sensor to image processing, the program has been able to view the sensing process as a whole. This has led to new developments in methods for band selection and analysis of the information content in spectral images.

Due to the large amount of collected data, the group had essentially processed data acquired in the visible domain, so far.

\subsection{Anomaly detection}

Anomaly detection is a conceptually simple method to detect targets in an image (Figure 3). This assumes 1) that the targets of interest make up a small fraction of the image, smaller than the amount of any constituent of the background, and 2) that the targets of interest exhibit a spectral contrast to the background. An anomaly detection algorithm then seeks to identify pixels which deviate from the background, and labels these as possible targets. This has the advantage that no prior information about the targets is needed; even unknown targets can be detected. On the other hand, if the background itself contains "anomalous" spectra, i.e. if assumption 1 is violated, false alarms will result. 

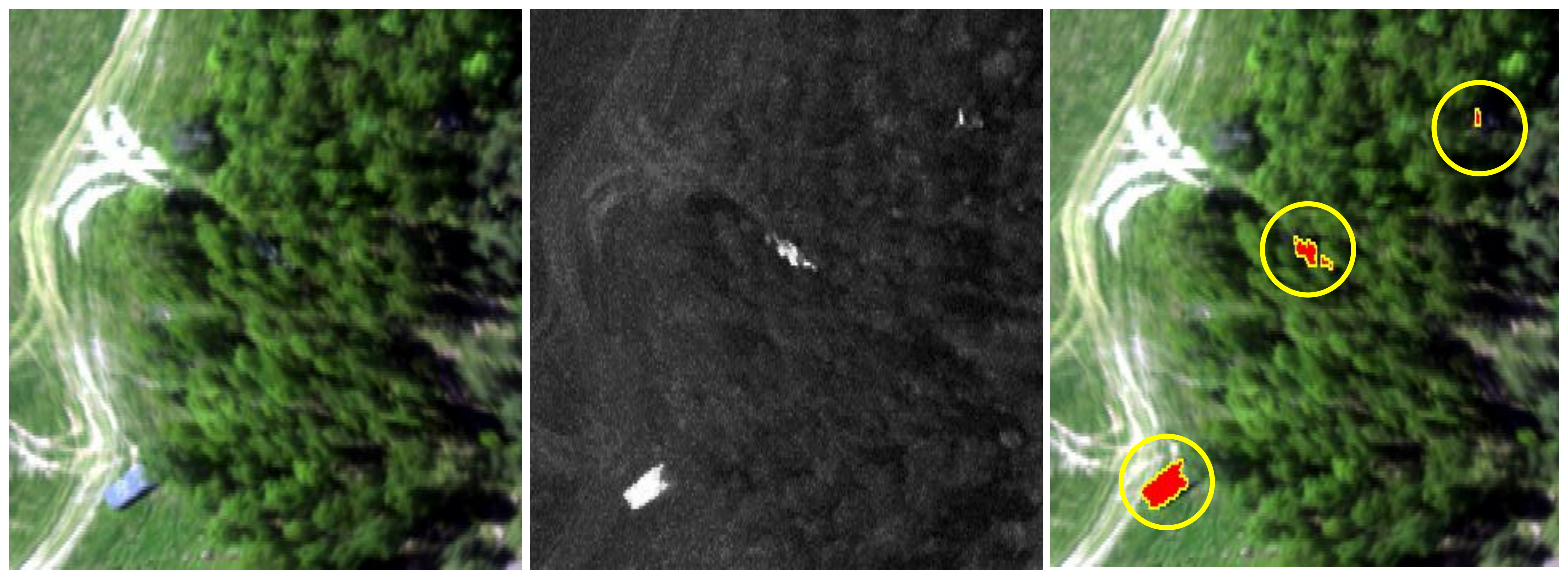

Figure 3: Hyperspectral processing example. The leftmost image is a visual colour image extracted from a hyperspectral image. The middle image shows the result from processing of the full spectral information to emphasise pixels that deviate from the background. This reveals the presence of two partially hidden targets. In the rightmost image, detected target pixels are highlighted. This is essentially a fully automatic process.

\subsection{Signature-based detection}

If the spectral characteristics of a target is known, signature-based detection, or spectral recognition, can be applied to an image to search for pixel spectra consistent with the known signature. The signature of a target is typically represented as a spectral reflectance (or emissivity) curve. The sensor, however, measures a radiance spectrum. This is influenced not only by the target reflectance, but also by atmospheric scattering and absorption, as well as illumination conditions at the target and, for thermal spectral imaging, also the target temperature. Thus a given spectral signature may give rise to a range of different radiance spectra at the sensor. Therefore, signature-based detection is not a straightforward task.

Using atmospheric propagation models, it is possible to convert the received radiance spectrum into an estimate of target reflectance in each pixel of an image. This can then be compared to the known reflectance signature of the target of interest. A similarity metric is calculated in each pixel and threshold to detect the targets matching the given signature.

An alternative approach in the case of such parameter uncertainties is to model the range, or subspace, of radiance spectra which could arise from the target reflectance signature under the range of possible atmospheric conditions and target geometries. If the received radiance spectrum is in this range, or close to it according to some similarity metric, it is considered a target. This "physical subspace" approach has the advantage that the algorithm can adapt to varying degrees of uncertainty in the model for received radiance from a target.

A simple case of signature-based detection occurs when the image contains a known reference target with the desired signature. In this case, it can usually be assumed that the atmospheric effects, and to some extent also the illumination, are the same for the reference targets and any unknown targets in the image. It is then possible to apply algorithms which simply compare the radiance spectra of all pixels in the image to the radiance of the reference target. Targets with spectra similar to the reference target can then be readily detected in many cases.

In the JP 8.10 program, these three classes of signature-based detection have been employed with generally good results. It has been shown that knowledge about target signatures generally improves the target detection probability. It is also possible, in many important cases, to discriminate between classes of targets. A priori signature information has been taken from reflectance spectra measured directly at the target using field spectrometers. It can be noted that an operational military spectral imaging system will most likely need to include functionality for extracting signature information from images ("spectral intelligence"). 


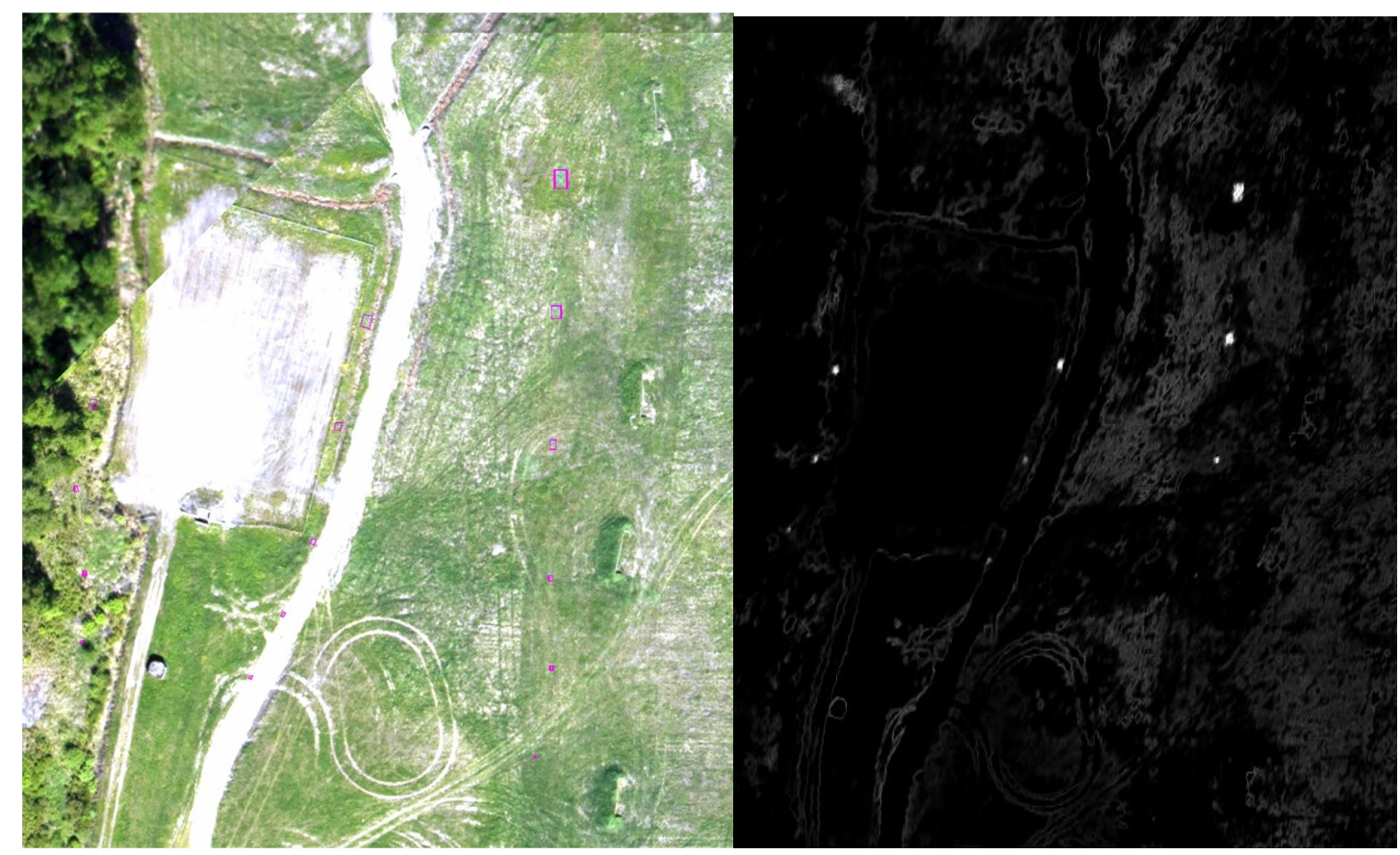

Figure 4: Result from signature-based detection in an image from the ASI sensor. To the left is a section of a mosaic of images from Kvarn recorded with a RGB digital camera with high spatial resolution. An array of varying size pieces of the same material (a civilian camouflage net) are marked with purple boxes. The targets' visual contrast is low, but they are readily detected in ASI hyperspectral data (right) based on an independently measured reflectance spectrum, using a physical subspace algorithm.

\subsection{Band selection}

If the number of spectral bands can be limited this can be advantageous for several reasons:

- By aggregating successive hyperspectral bands, the signal to noise ratio for each of the resulting multi spectral bands is larger than for each corresponding hyperspectral bands

- The amount of data generated and therefore the data transfer rate is lowered

- The equipment cost can be reduced if the number of bands is limited to less than five

- A simpler DRI algorithm can be used

- The time delay between recording a hyper/multicube and generating an alarm is reduced

The band parameters which then have to be determined as function of scenario are the number of bands required, their spectral position and their bandwidth.

A band selection algorithm has been developed that finds optimum band positions using a discriminant analysis technique based on a Mahalanobis distance measure taking into account the statistical variation in target and background pixels in the training set.

Questions that have to be answered are:

- How robust are the band parameters for time of day, time of year, weather, ...?

- How dependent are the band parameters on scenario?

- How dependent are the band parameters on the target and background training set?

Initial results, based on radiance spectra analysis, have shown that there is not a single unique band combination in the LWIR for classification. Further works are necessary to consolidate these results taking into account the atmosphere compensation and the temperature - emissivity separation.

\section{Conclusion}

The huge amount of data acquired during the different trials allows us to demonstrate the gain brought by spectral imaging for military applications. Although a large optical properties data base was generated and proved useful, 
additional data of other environments, like urban, desert ..., and other man-made materials should be acquired in future studies. Thereby, the set of material parameters should also be increased to a complete set of spectral directional properties and additional properties like thermal parameters and biophysical parameters.

Phenomenology studies have been conducted to analyze and validate the spectral information in the understanding of an optronic landscape. The main results point out how the spectral signature impacts at sensor level signal, but also highlight the role of the vegetation transpiration and the surface contaminant on the spectral signature. The separability is consistent with anomaly detection in the reflective domain but, in addition, in the infrared domain atmospheric compensation and temperature-emissivity separation algorithms are required.

Furthermore, work on the validation of an end-to-end simulation tool (Cameo-Sim) has been conducted, and then used to extend the evaluation of the tested military scenarios. First validation results, based on available data and several evaluation criteria, exhibit encouraging results. Nevertheless, the validation process must be pursued with more extensive data sets.

To evaluate the utility of spectral imaging for defense applications, several tactical scenarios have been tested. This required the development of spectral image processing tools such as anomaly detection and signature based discrimination. The results shows that spectral imaging brings strong support for automatic anomaly detection, especially when the shape of the target is not resolved. The robustness of the different spectral processing techniques is also demonstrated when comparing the results from on-ground, airborne acquisitions and synthetic images.

For each analyzed tactical scenario, the spectral imaging exhibits very good performances for automatic anomaly detection and recognition. It was difficult to compare the performance of an up-to-date high spatial resolution broadband image sensor with a spectral image sensor. The reason is that the group did not have access to up-to-date spatial image processing capabilities with proven automatic detection and classification performance. Hyperspectral imaging generates an amount of information per pixel which is larger than any other imaging technique. In principle, any image processing task should be made easier by a larger amount of information. However, spectral image data beyond 3 bands cannot be easily visualized for a human image interpreter, which is still the gold standard for conventional spatial image processing. Automatic processing by computer is therefore an integral part of the hyperspectral imaging process; much more so than for conventional imaging. It must be noted that there is a fundamental tradeoff between spectral and spatial resolution due to the finite number of photons available. A single pixel in a spectral image with 100 bands would have a signal-to-noise ratio comparable to that of a 10x10 pixel region in a conventional image. The amount of data is also about the same, and one could envisage in both cases that the data would be sufficient for, say, recognition of a type of vehicles. The same could hold for in-between cases with a different mix of spectral and spatial information. This observation suggests that it may not be straightforward to define an optimal compromise between spectral and spatial resolution in a spectral imaging system.

Overall, the progress within THALES JP8.10 has been substantial. A significant military potential of spectral imaging has been demonstrated. Recommendations can be made regarding visible and near-infrared sensors. The collaboration continues under the EUROPA HIPOD program, which aims to extend the analysis of existing data with emphasis on spectral imaging in the thermal infrared.

This work has been funded by the United Kingdom, Netherlands, French, German, Italian, Norwegian and Sweden MoD's in the context of the European Defense R\&T Cooperation. 